\title{
Hipertensión arterial: el factor de riesgo más importante para grosor íntima-media carotídeo elevado y placa carotídea en adultos de Santiago
}

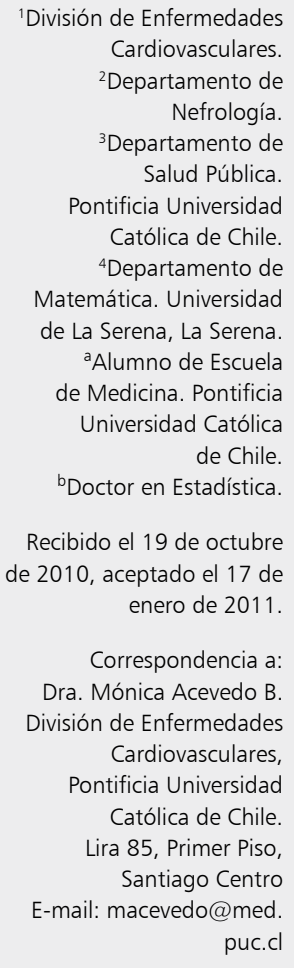

'División de Enfermedades Cardiovasculares. ${ }^{2}$ Departamento de Nefrología. ${ }^{3}$ Departamento de Salud Pública. Pontificia Universidad Católica de Chile. ${ }^{4}$ Departamento de Matemática. Universidad de La Serena, La Serena. aAlumno de Escuela de Medicina. Pontificia Universidad Católica de Chile.

${ }^{b}$ Doctor en Estadística.

Recibido el 19 de octubre de 2010, aceptado el 17 de enero de 2011.

Correspondencia a: Dra. Mónica Acevedo B. División de Enfermedades Cardiovasculares,

Pontificia Universidad Católica de Chile.

Lira 85, Primer Piso, Santiago Centro

E-mail:macevedo@med. puc.cl

\author{
MÓNICA ACEVEDO ${ }^{1}$, RODRIGO TAGLE ${ }^{2}$, VERÓNICA KRAMER ${ }^{1}$, \\ PILAR ARNAÍZ' ${ }^{1}$, ARNALDO MARÍN ${ }^{a}$, FELIPE PINO ${ }^{a}$, IVÁN GODOY ${ }^{1}$, \\ XIMENA BERRÍOS ${ }^{3}$, CARLOS NAVARRETE $^{4,6}$
}

\section{Risk factors for a high carotid intima media thickness among healthy adults}

Background: Carotid intima media thickness (CIMT) is a marker of cardiovascular damage that can be modified by traditional risk factors. Aim: To determine attributable risk factors for a high CIMT among healthy adults. Material and Methods: A sample of 1270 individuals (636 males and 634 females) aged $44 \pm 11$ years, was studied. Blood pressure, weight, height, lipid profile and blood glucose were measured in all. CIMT and the presence of atherosclerotic plaques were determined by carotid ultrasound. Standard criteria were used to define hypertension, dyslipidemia and diabetes. Results: Mean CIMT in the sample studied was $0.62 \pm 0.01 \mathrm{~mm}$ and percentile 75 was 0.67 . The most important risk factor for a CIMT over percentile 75 and the presence of atherosclerotic plaques was hypertension with attributable risks of 54 and 57\%, respectively. Conclusions: In this sample, the main risk factor for a high CIMT was hypertension.

(Rev Med Chile 2011; 139: 290-297).

Key words: Carotid artery diseases; Hypertension; Risk factors.
E 1 grosor íntima-media carotídeo (carotid intima media thickness: CIMT) es un marcador de aterosclerosis subclínica. Es medido por ultrasonografía y es un método seguro, no invasivo y reproducible ${ }^{1}$. Numerosos estudios han demostrado que el CIMT se asocia a los factores de riesgo (FR) cardiovascular $(\mathrm{CV})$ tradiciona$\operatorname{les}^{2-4}$ y se modifica según su carga, y representa el resultado de la respuesta de cada persona a su propia carga de $\mathrm{FR}^{5}$. Asimismo, varía según el grupo étnico. Los valores promedio en población general en Latinoamérica, incluyendo Santiago, han sido recientemente publicados ${ }^{6}$. En adultos chilenos hemos reportado que el CIMT y las placas carotídeas aumentan según la carga de $\mathrm{FR}^{7}$.

La importancia del CIMT es que su aumento marca la presencia de ateroesclerosis y se asocia a eventos $\mathrm{CV}$, aun después de ajustar por los FR tradicionales ${ }^{8,9}$. Este marcador acaba de ser aceptado por la Sociedad Americana y el Colegio Americano de Cardiología (AHA/ACC) en la evaluación del riesgo cardiovascular en sujetos asintomáticos con un alto nivel de evidencia ${ }^{10}$.

El estudio INTERHEART-Latin America ${ }^{11}$ demostró que en sujetos que habían tenido un infarto al miocardio, el FR atribuible poblacional más importante para el infarto era la obesidad abdominal, a diferencia del resto del mundo, en que fue la dislipidemia. Los hallazgos de este estudio destacaron que los FR determinantes de infarto al miocardio eran diferentes en distintas áreas del mundo. Lanas y cols ${ }^{12}$, posteriormente, en el estudio INTERHEART-Chile demostraron que a diferencia de Latinoamérica y el mundo, 
el FR atribuible más importante para infarto en nuestro país era el tabaquismo.

Motivados por estos resultados, quisimos investigar si existían datos internacionales que reportaran sobre la influencia de los distintos FR en el grosor de la carótida. El conocimiento de qué factores son los más importantes en el incremento de este biomarcador intermedio, CIMT, podría ayudarnos a seleccionar mejor a la población, en que este marcador pueda ser usado para mejorar la predicción del riesgo $\mathrm{CV}$.

El objetivo de este estudio fue entonces, investigar qué FR tradicionales o emergentes determinan un CIMT elevado o presencia de placa carotídea en adultos de Santiago.

\section{Métodos}

Estudio transversal realizado en población urbana de Santiago entre los años 2006 y 2007, por muestreo epidemiológico estratificado en adultos entre 20 y 65 años de 3 estratos socio-económicos: bajo, medio-bajo y medio-alto. Se excluyeron aquellos sujetos con antecedente de enfermedad aterosclerótica, infección reciente, o enfermedades inflamatorias no infecciosas conocidas, y aquellos con proteína C-reactiva ultrasensible (PCRus) $>10 \mathrm{mg} / \mathrm{L}$, sugerente de infección.

\section{Recolección de Datos}

Los sujetos fueron encuestados en sus hogares. La encuesta incluía antecedentes demográficos y médicos previos, factores de riesgo CV y medicamentos. Luego, todos fueron citados al centro universitario, donde una enfermera les midió peso, talla, índice de masa corporal (IMC), cintura, cadera, y presión arterial sistólica y diastólica (PAS-PAD) en reposo, en 3 oportunidades y en 1 sólo día, según recomendaciones de JNC 7, y les realizó extracción de sangre venosa en ayunas para determinación de perfil lipídico, glicemia y PCRus. Todos los sujetos fueron citados nuevamente, en el plazo de 1 semana, al centro universitario, para el examen físico y determinación del CIMT.

Se consideró hipertensos a todos los sujetos con diagnóstico médico de HTA, con o sin fármacos y a aquellos con más de $2 \mathrm{PA} \geq 140 / 90$ mmHg; se definió pre-hipertensión arterial como la PAS de 120 a 139 y/o PAD de 80 a 89 mmHg en el examen actual; consideramos dislipidémicos a aquellos sujetos con diagnóstico de hipercolesterolemia diagnosticada por médico, con o sin tratamiento farmacológico, y a aquellos con colesterol total $>200 \mathrm{mg} / \mathrm{dL}$, y/o colesterol HDL $<40$ en hombres y $<50 \mathrm{mg} / \mathrm{L}$ en mujeres en el examen actual. Diabetes fue considerada como positiva en aquellos con diagnóstico médico de diabetes con o sin medicamentos, y aquellos con glicemia de ayuno mayor de $126 \mathrm{mg} / \mathrm{dL}$. Se consideró historia familiar de cardiopatía coronaria cuando había historia de familiar directo hombre $<55$ o mujer $<65$ años con este antecedente en la familia.

Todos los sujetos firmaron un consentimiento informado para participar en el estudio, aprobado por el comité de Ética de la universidad ejecutora.

\section{Mediciones de laboratorio}

Las muestras para perfil lipídico, glicemia y PCRus fueron obtenidas por punción venosa, con ayuno de 12 horas.

a) Colesterol total, colesterol HDL y triglicéridos: Fueron medidos usando los métodos enzimáticos estándar con reactivos ad-hoc (analizador Yací).

b) Colesterol LDL: Fue calculado por fórmula de Friedewald.

c) Glicemia: Método glucosa oxidasa.

d) PCRus: Método nefelométrico (Dade Behring BN II; límite de detección 0,1 mg/L). Para el análisis estadístico se usó un valor de corte de PCRus $>2 \mathrm{mg} / \mathrm{L}$, sugerido por $\mathrm{CDC} / \mathrm{AHA}^{13}$ en el año 2003 como valor elevado de este marcador.

\section{Medición de Grosor Íntima-Media Carotídeo (CIMT)}

Se realizó según las recomendaciones del consenso de Manheim ${ }^{13}$. Para la medición del CIMT y placa se utilizó un transductor lineal de alta frecuencia (5-13 Mhz). La imagen se focalizó en la pared posterior de cada arteria carótida común, en áreas sin placas, donde se eligió un segmento de $1 \mathrm{~cm}$, proximal a la bifurcación carotídea de cada lado. Se realizaron mediciones en cada carótida común, en 3 ventanas ecográficas, utilizando el software $\mathrm{M}^{\prime}$ Ath ${ }^{\circledR}$. Todas las mediciones se realizaron "off-line” y en fin de diástole. Placa carotídea se definió como una protrusión en el lumen mayor al 50\% del CIMT adyacente o CIMT > 1,5 mm. El valor de CIMT registrado correspondió al mayor valor de CIMT medio entre el lado derecho e iz- 
quierdo. Las mediciones fueron ciegas al resultado de los exámenes de sangre. Para efectos de análisis estadístico, se utilizó sólo CIMT medio máximo. El coeficiente de variación intraoperador de nuestro grupo es de $3,8 \%$.

\section{Estadística}

Para las comparaciones de promedios se ocupó análisis de varianza. Los resultados se expresan como promedio (desviación estándar [DE]) o promedio $\pm \mathrm{DE}$. La PCRus se analizó en escala logarítmica para corregir asimetría de la distribución. Los odds ratios (OR) se calcularon en base de modelos de regresión logística múltiple, ajustados por edad y sexo, y se expresan por OR (IC 95\%). Se utilizó OR como aproximación al riesgo relativo (RR) para el cálculo del riesgo atribuible (RA), estimado a su vez como RA $=(\mathrm{RR}-1) / \mathrm{RR}$. Todos los cálculos se realizaron ocupando el software estadístico R.

\section{Resultados}

Se estudiaron 1.270 sujetos (636 hombres y 634 mujeres). La edad promedio del grupo fue $44 \pm 11$ años.

La prevalencia de HTA fue de $30 \%$, prehipertensión $8,9 \%$, dislipidemia $69 \%$, diabetes $13 \%$, tabaquismo activo $46 \%$. La prevalencia de síndrome metabólico (según ATP III) fue de $25 \%$. En la Tabla 1 se muestran algunas variables demográficas, las prevalencias de los FR y los valores de colesterol total, LDL y HDL, triglicéridos, glicemia, PAS y PAD, PCRus y el valor promedio de CIMT medio máximo, según sexo. Como se aprecia en la Tabla 1, la prevalencia de dislipidemia, antecedente familiar de cardiopatía coronaria, síndrome metabólico, IMC > 27 y PCRus fueron significativamente mayores en mujeres que en hombres. La prevalencia de HTA fue significativamente mayor en hombres que en mujeres.

Tabla 1. Variables demográficas, prevalencia de FR cardiovascular, medidas antropométricas, perfil lipídico, proteína c-reactiva y grosor íntima-media carotídeo en la población estudiada, por sexo. Valores expresados como promedio (desviación estándar) o porcentaje

\begin{tabular}{|c|c|c|c|c|}
\hline & $\begin{array}{l}\text { Hombres } \\
(n=636)\end{array}$ & $\begin{array}{l}\text { Mujeres } \\
(n=634)\end{array}$ & $\begin{array}{c}\text { Total } \\
(1.270)\end{array}$ & $\mathbf{p}$ \\
\hline Edad (años) & $44 \quad(11)$ & $44 \quad(11)$ & $44 \quad(11)$ & NS \\
\hline Hipertensión arterial (\%) & 33 & 28 & 30 & 0,039 \\
\hline Dislipidemia (\%) & 64 & 75 & 69 & $<0,001$ \\
\hline Diabetes (\%) & 14 & 12 & 13 & NS \\
\hline Tabaquismo (\%) & 48 & 44 & 46 & NS \\
\hline Historia familiar de cardiopatía coronaria & 15 & 20 & 18 & $<0,01$ \\
\hline S. metabólico (\%) & 21 & 28 & 25 & $<0,01$ \\
\hline IMC $\left(\mathrm{kg} / \mathrm{m}^{2}\right)$ & $28 \quad(4)$ & (6) & $(5)$ & 0,013 \\
\hline $\mathrm{IMC}>27(\%)$ & 52 & 55 & 53 & NS \\
\hline Cintura $(\mathrm{cm})$ & $94 \quad(10)$ & $89(12)$ & $91 \quad(11)$ & $<0,0001$ \\
\hline Colesterol total (mg/L) & $201 \quad(40)$ & $204(43)$ & $203(42)$ & NS \\
\hline Colesterol HDL (mg/L) & $46 \quad(11)$ & $53 \quad(13)$ & 49 (13) & $<0,001$ \\
\hline Colesterol LDL (mg/L) & $121 \quad(34)$ & 122 (33) & 121 & NS \\
\hline Triglicéridos (mg/L) & $176(119)$ & $140(100)$ & $158(112)$ & $<0,001$ \\
\hline Presión arterial sistólica $(\mathrm{mmHg})$ & $124(20)$ & $119(21)$ & $122(20)$ & $<0,001$ \\
\hline Presión arterial diastólica $(\mathrm{mmHg})$ & $82(12)$ & $77 \quad(12)$ & $79(12)$ & $<0,001$ \\
\hline CIMT medio máximo $(\mathrm{mm})$ & $0,63 \quad(0,01)$ & $(0,01)$ & $0,62 \quad(0,01)$ & $<0,001$ \\
\hline PCRus (mg/L) & $1,92 \quad(1,99)$ & $(2,47)$ & $(2,27)$ & $<0,001$ \\
\hline
\end{tabular}

IMC = índice de masa corporal; CIMT = grosor íntima-media carotídeo. 
El CIMT medio promedio fue $0,62 \pm$ $0,01 \mathrm{~mm}$. En hombres, el CIMT medio fue de 0,63 $\pm 0,01 \mathrm{~mm}$ y en mujeres de 0,60 \pm $0,01 \mathrm{~mm}$, con diferencia significativa entre ambos géneros. Se detectó presencia de placa aterosclerótica en 14\% de los sujetos, sin presentarse diferencias por sexo. El CIMT se correlacionó en forma directa y significativa con la edad, IMC, cintura, PAS y PAD, colesterol total, LDL, PCRus y triglicéridos, e inversamente con el colesterol HDL (Tabla 2).

Para definir el riesgo de CIMT elevado, se utilizó el CIMT mayor al percentil 75 de la población en estudio. Este valor fue de $0,67 \mathrm{~mm}$. El utilizar el valor de CIMT mayor al percentil 75 fue sugerido por el grupo SHAPE (Screening for Heart Attack Prevention and Education Task Force report ${ }^{16}$ para definir a sujetos de mayor riesgo de eventos CV cuando se usa CIMT.

En la Tabla 3 se presenta el odds ratio y el riesgo atribuible porcentual para cada factor de riesgo, ajustado por edad y sexo. El FR más importante para CIMT elevado fue la HTA con un OR $=2,17$ y un riesgo atribuible de $54 \%$. Este fue seguido por la pre-HTA con un OR = 1,59 y RA de $37 \%$, el IMC > 27 con OR $=1,55$ y RA de $36 \%$ y, finalmente, la cintura ( $>102 / 88 \mathrm{~cm}$, hombres y mujeres, respectivamente) con un OR $=1,4$ (RA 28\%) y la PCRus, con un OR $=1,32$ (RA 24\%). Estos 2 últimos factores presentaron sólo discreta significancia estadística (Tabla 3). Los OR para dislipidemia, historia familiar de infarto, tabaquismo y diabetes no fueron estadísticamente significativos. Asimismo, también incluimos como variables el colesterol LDL > 160 $\mathrm{mg} / \mathrm{dL}$ (valor considerado alto según ATPIII) y triglicéridos $\geq 150 \mathrm{mg} / \mathrm{dL}$ (también considerado como elevado según ATPIII). Ninguno de estas variables fue determinante significativo de CIMT. Cuando incluimos como otra variable independiente el síndrome metabólico, este sí fue un determinante significativo de CIMT elevado $(\mathrm{OR}=1,54$; 1,11-2,13, RA 35\%). El análisis para edad como FR de CIMT elevado, también fue significativo (ajustado sólo por sexo): OR $=1,14$ y RA $12 \%$ (Tabla 3 ).
Tabla 2. Correlaciones de Pearson (R) parciales, ajustadas por edad y sexo entre grosor íntima-media carotídeo y distintos parámetros antropométricos, bioquímicos, presión arterial y proteína C-reactiva ultrasensible

\begin{tabular}{|lcc|}
\hline & R & p \\
Edad & 0,59 & $<0,0001$ \\
IMC & 0,18 & $<0,0001$ \\
Circunferencia cintura & 0,16 & $<0,0001$ \\
Presión arterial sistólica & 0,28 & $<0,0001$ \\
Presión arterial diastólica & 0,20 & $<0,0001$ \\
Colesterol total & 0,12 & $<0,001$ \\
Triglicéridos & 0,10 & $<0,01$ \\
Glicemia & $-0,05$ & $\mathrm{NS}$ \\
Colesterol LDL & 0,12 & $<0,001$ \\
Colesterol HDL & $-0,11$ & $<0,001$ \\
log (PCRus) & 0,25 & $<0,001$ \\
\hline
\end{tabular}

$\mathrm{IMC}=$ índice de masa corporal.

Tabla 3. Riesgo atribuible para grosor íntima-media carotídeo elevado ( $>$ al percentil 75), ajustado por edad y sexo

\begin{tabular}{|lccc|}
\hline Variable & OR & IC & RA \% \\
Edad* & 1,14 & $(1,12-1,15)$ & 12 \\
HTA & 2,17 & $(1,59-2,95)$ & 54 \\
Pre-HTA & 1,59 & $(1,16-2,16)$ & 37 \\
IMC $>27$ & 1,55 & $(1,14-2,10)$ & 36 \\
\hline $\begin{array}{l}\text { Cintura ( > 102/88 cm } \\
\text { hombres/mujeres) }\end{array}$ & 1,4 & $(1,00-1,94)$ & 28 \\
PCRus > 2 mg/L & 1,32 & $(1,00-1,80)$ & 24 \\
\hline $\begin{array}{l}\text { Síndrome metabólico (ATP III) } \\
\text { Dislipidemia }\end{array}$ & 1,54 & $(1,11-2,13)$ & 35 \\
Colesterol LDL > 160 mg/dL & 1,18 & $(0,84-1,66)$ & 15 \\
\hline Triglicéridos $\geq 150$ mg/dL & 1,29 & $(0,86-1,94)$ & 23 \\
\hline $\begin{array}{l}\text { H. familiar cardiopatía } \\
\text { coronaria }\end{array}$ & 1,11 & $(0,77-1,62)$ & 10 \\
\hline Tabaquismo & 1,02 & $(0,75-1,37)$ & 2 \\
\hline Diabetes & 1,01 & $(0,67-1,51)$ & $<1,0$ \\
\hline
\end{tabular}

$\mathrm{RA}=$ riesgo atribuible; $\mathrm{RR}=$ riesgo relativo; $\mathrm{OR}=$ odds ratio; $\mathrm{IC}=$ intervalo de confianza; HTA = hipertensión arterial; * = sólo ajustado por sexo. $R A=$ Riesgo atribuible $=(R R-1 / R R)$. Los $R R$ están aproximados por OR. 
Tabla 4. Odds Ratio para CIMT elevado ( $>$ al percentil 75), ajustado por edad y sexo, para variables continuas

\begin{tabular}{|llc|}
\hline Variable & OR & IC \\
\hline Presión arterial sistólica & 1,03 & $(1,02-1,04)$ \\
\hline Presión de pulso (PAS-PAD) & 1,04 & $(1,02-1,05)$ \\
\hline Presión arterial diastólica & 1,03 & $(1,02-1,04)$ \\
IMC & 1,06 & $(1,03-1,10)$ \\
Colesterol total & 1 & $(1,00-1,01)$ \\
Triglicéridos & 1 & $(1,00-1,01)$ \\
Colesterol HDL & 0,99 & $(0,97-1,00)$ \\
Colesterol LDL & 1 & $(1,00-1,01)$ \\
Glicemia en ayuna & 1 & $(1,00-1,01)$ \\
\hline
\end{tabular}

En forma continua y ajustadas por edad y sexo, las variables asociadas en forma significativa con CIMT elevado fueron PAS, PAD, presión de pulso e IMC (Tabla 4).

Finalmente, modelados en forma conjunta (análisis de regresión logística múltiple) y ajustados por edad y sexo, también se demostró que el factor más importante en determinar CIMT elevado fue la HTA (OR = 2,14; IC: 1,54-2,97). Otros determinantes, también significativos, fueron el sexo masculino, la edad, y el IMC > 27 (Tabla 5).

Para placa aterosclerótica carotídea, el único determinante significativo, ajustado por edad y sexo, fue la HTA con OR = 2,31 (IC: 1,61-3,32) y con RA $=57 \%$. Ninguno de los otros FR estudiados fue significativo en la determinación de placa (Tabla 6).

\section{Discusión}

Este estudio demuestra que, en la población estudiada de Santiago urbano, el CIMT elevado (CIMT > percentil 75) y la presencia de placas carotídeas son explicados, principalmente, por la HTA. Otros FR importantes, pero que sólo determinaron un CIMT elevado, pero no placa, fueron la edad, IMC $>27$ y el sexo masculino.

Numerosos estudios han confirmado que el CIMT aumenta según el número de FR tradicionales ${ }^{1-3,5}$. Estos hallazgos nos alertaron en cuanto a poder distinguir subgrupos, dentro de la población general, que se pudieran beneficiar más
Tabla 5. Modelo de regresión logística múltiple para grosor medio carotídeo elevado (mayor a 0,67 mm)

\begin{tabular}{|lcl|}
\hline \multicolumn{1}{c}{ Variable } & OR & \multicolumn{1}{c|}{ IC } \\
\hline Hipertensión arterial & 2,14 & $1,54-2,97$ \\
\hline Edad & 1,12 & $1,10-1,14$ \\
Sexo masculino & 1,47 & $1,08-2,0$ \\
\hline IMC $>$ 27 & 1,44 & $1,04-2,01$ \\
\hline Dislipidemia & 1,05 & $0,73-1,5$ \\
\hline Diabetes & 0,86 & $0,56-1,33$ \\
\hline Tabaquismo & 1,10 & $0,80-1,51$ \\
PCRus $>2$ mg/L & 1,10 & $0,79-1,54$ \\
\hline
\end{tabular}

Tabla 6. Riesgo atribuible para placa, ajustado por edad y sexo

\begin{tabular}{|lccc|}
\hline Variable & \multicolumn{1}{c}{ OR (IC) } & RA (\%) \\
Diabetes & 1,29 & $(0,82-2,01)$ & 22 \\
Tabaquismo & 1,35 & $(0,95-1,92)$ & 26 \\
\hline HTA & 2,31 & $(1,61-3,32)$ & 57 \\
\hline H. familiar CC & 1,21 & $(0,79-1,85)$ & 17 \\
\hline PCRus $>2$ & 1,24 & $(0,86-1,79)$ & 19 \\
Dislipidemia & 1,31 & $(0,86-2,0)$ & 24 \\
\hline IMC > 27 & 0,93 & $(0,65-1,32)$ & $<1$ \\
\hline
\end{tabular}

$\mathrm{RA}=$ riesgo atribuible; $\mathrm{RR}=$ riesgo relativo; $\mathrm{OR}=$ odds ratio; $\mathrm{IC}=$ intervalo de confianza. RA $=(\mathrm{RR}-1 / \mathrm{RR})$. Los RR están aproximados por OR.

del uso de este marcador para la estratificación del riesgo CV. Por el otro lado, dado que varios estudios de intervención tanto farmacológicos (con antihipertensivos ${ }^{14}$ y estatinas ${ }^{15}$ ), como de estilos de vida (con dieta y ejercicio ${ }^{16}$ ) habían demostrado que el CIMT podía disminuir con el tratamiento agresivo del factor de riesgo, el conocer cuáles eran los factores que más influían sobre este marcador en nuestra población nos pareció fundamental. Lanas y $\operatorname{cols}^{11}$, primero en el estudio INTERHEART-Latin America, y posteriormente, en el INTERHEART-Chile ${ }^{12}$, nos sorprendieron al reportar que en sujetos con infarto al miocardio los FR atribuibles más importantes eran distintos en las distintas regiones del mundo. En Chile, a diferencia del resto del mundo y de Latinoamérica, los factores más determinantes de infarto fueron 
el tabaquismo y después la dislipidemia y la HTA. Es decir, las distintas poblaciones en el mundo respondieron en forma distinta a los distintos FR. Esta fue la principal motivación de nuestro estudio: buscar qué factores influirían más en el CIMT y presencia de placa carotídea en nuestra población.

El FR más importante, tanto para CIMT elevado, como para placa carotídea en nuestra población, fue la HTA. Existen numerosas publicaciones que confirman la HTA como un factor asociado al incremento del grosor carotídeo y la aterosclerosis carotídea propiamente tal ${ }^{17-19}$. Incluso, un trabajo reciente demostró que la pre-hipertensión producía aumento del grosor carotídeo y placas carotídeas en comparación a sujetos normoten$\operatorname{sos}^{20}$. Sin embargo, la mayoría de los estudios, no se han realizado en población general, sino que en poblaciones de hipertensos versus normotensos, lo que no nos permite hacer comparaciones directas en cuanto a odds ratios. En nuestro estudio, tanto en el análisis de riesgo atribuible, como en el modelo de regresión múltiple, la HTA fue el factor determinante más importante de aterosclerosis subclínica. Asimismo, también encontramos que la pre-hipertensión determinaba mayor CIMT. $\mathrm{Su}$ y $\mathrm{cols}^{17}$ también reportaron que la HTA era el factor más importante en determinar un CIMT elevado. Lo seguían, la edad y el tabaquismo. Sin embargo, en ese reporte los autores seleccionaron a una población ya hipertensa y la compararon con una normotensa. A pesar de sus limitaciones, el estudio de $\mathrm{Su}$ y cols concuerda con nuestros hallazgos de que, en distintas poblaciones, los factores determinantes de aterosclerosis subclínica carotídea pueden ser diferentes. Cabe destacar, sin embargo, que dado que nuestro estudio es de corte transversal no se puede saber si este hallazgo es por caracteres propios de la población chilena o por otros factores que pudieran estar involucrados en la cadena causal.

Otro artículo, en niños y jóvenes canadienses, demostró que el FR más importante en determinar CIMT elevado era la edad, seguido por la dislipidemia y luego por el antecedente de enfermedad coronaria prematura ${ }^{21}$. Si bien este último artículo persiguió objetivos parecidos a los nuestros, los sujetos seleccionados no fueron de la población general, sino de un grupo de niños y jóvenes con hiperlipidemia ya establecida y otro grupo de obesos. En este último estudio, sin embargo, la HTA no fue un determinante importante de aterosclerosis carotídea. Yan y cols ${ }^{22}$, en una población adulta canadiense, pero sólo de hombres, estudiaron la relación entre distintas variables de obesidad y grosor carotídeo. En el análisis de regresión múltiple, la PA sistólica y el tabaquismo fueron los determinantes más importantes, seguidos de la relación cintura/cadera. De nuevo, los FR determinantes de aterosclerosis carotídea en esa población, fueron diferentes a los de la nuestra.

La HTA es un FR muy importante para el desarrollo de eventos cardio y cerebrovasculares. Actualmente, las guías europeas de HTA aconsejan la realización de una ecografía carotídea con medición de CIMT cuando se requiere de una mejor evaluación del riesgo $\mathrm{CV}$ en pacientes hipertensos ${ }^{23}$. Recientemente, Zanchetti y cols $^{24}$ confirmaron la utilidad de esto: en pacientes hipertensos demostraron que el CIMT elevado agregaba valor pronóstico en los eventos $\mathrm{CV}$, independiente del tratamiento antihipertensivo y de su duración y de los otros FR. En nuestro país no existen guías con una posición sólida en este respecto. Los resultados aquí presentados, en población adulta de Santiago, dejan claro que este biomarcador podría ser útil en la mejor caracterización del riesgo de eventos CV en hipertensos. En este sentido, un artículo reciente, que relaciona positivamente el CIMT elevado y la presencia de PCRus elevada (es decir de inflamación subclínica) con la incidencia de hospitalizaciones por insuficiencia cardiaca propone que, a diferencia de un factor como la presión arterial, que puede variar mucho en un mismo individuo, el "CIMT refleja la exposición del mismo sujeto, durante un período más largo de tiempo, al factor de riesgo", lo que le daría al CIMT un alto y sólido valor pronóstico ${ }^{25,26}$.

El IMC > 27 también fue un determinante importante de CIMT elevado en nuestro estudio. Este hallazgo también ha sido descrito por Burke y $\operatorname{cols}^{27}$. Ellos demostraron que la obesidad daba un mayor riesgo de CIMT elevado. Previo a estos resultados, había sólo 2 reportes aislados, ambos en niños y adolescentes, que habían asociado el CIMT con el sobrepeso y obesidad, y sus resultados habían sido discordantes ${ }^{28,29}$. Nuestro estudio, no sólo demostró que el IMC $>27$ daba un mayor riesgo de CIMT elevado, sino que este fue independiente de otros FR y de HTA (Tabla 5). Los resultados del estudio Burke y cols $^{27}$ en Estados Unidos de Norteamérica produjeron alerta sobre el eventual 
aumento en el riesgo de eventos CV futuros en la población con sobrepeso/obesidad basados en el uso del CIMT como predictor de eventos.

En nuestros resultados llama la atención que FR tan importantes, como la dislipidemia, diabetes y tabaquismo no hayan sido determinantes significativos de CIMT o placa carotídea. Incluso usando niveles de corte altos para LDL (>160 mg/ $\mathrm{dL}$ ), este factor no fue determinante de CIMT elevado ni placa. Algunos estudios han demostrado que el daño estructural en la arteria, en este caso la carótida, se daría sólo en personas que, además de tener el colesterol elevado, tienen al mismo tiempo, mayores rangos de presión arterial. Es en ellas en que se produciría un daño aterosclerótico más acelerado, como lo señala Sun y $\operatorname{cols}^{30}$. Estos resultados ameritan una investigación futura.

Finalmente, la PCRus no fue un marcador significativo de aumento de CIMT. Algunos estudios han asociado la PCRus a la progresión del CIMT, pero otros, en grandes poblaciones, no han podido replicar esos resultados ${ }^{31,32}$. Estudios previos de nuestro grupo, pero en niños, nos sugirieron que la PCRus estaría relacionada al CIMT, pero probablemente a través de la obesidad general y abdominal ${ }^{33,34}$, lo que también se sugiere en este estudio, en que el IMC $>27$ fue determinante de CIMT, y la PCRus se perdió como factor al ajustar por otras variables.

\section{Limitaciones}

Es un estudio transversal, donde no se puede hablar de causalidad. La población estudiada es relativamente joven, sin adultos $>70$ años. La presión arterial fue tomada en un sólo día. Si bien se realizó ajuste por medicamentos hipolipemiantes y antihipertensivos, no se registró dosis. No se midieron otros FR no tradicionales, como por ejemplo creatinina sérica ni microalbuminuria, factores relacionados al daño cardiovascular y por HTA. Finalmente, no reportamos sobre seguimiento y eventos $\mathrm{CV}$.

\section{Referencias}

1. Chambless LE, Heiss G, Folsom AR, Rosamond W, Szklo $\mathrm{M}$, Sharrett AR, et al. Association of coronary heart disease incidence with carotid arterial wall thickness and major risk factors: The Atherosclerosis Risk in Communities (ARIC) study, 1987-1993. Am J Epidemiol 1997; 146: 483-94.
2. Mannami T, Konishi M, Baba S, Nishi N, Terao A. Prevalence of asymptomatic carotid atherosclerotic lesions detected by high-resolution ultrasonography and its relation to cardiovascular risk factors in the general population of a Japanese city: The Suita study. Stroke 1997; 28: 518-25.

3. O'Leary DH, Polak JF, Kronmal RA, Kittner SJ, Bond MG, Wolfson SK Jr, et al. Distribution and correlates of sonographically detected carotid artery disease in the cardiovascular health study. The CHS collaborative research group. Stroke 1992; 23: 1752-60.

4. Poli A, Tremoli E, Colombo A, Sirtori M, Pignoli P, Paoletti R. Ultrasonographic measurement of the common carotid artery wall thickness in hypercholesterolemic patients. A new model for the quantitation and follow-up of preclinical atherosclerosis in living human subjects. Atherosclerosis 1988; 70: 253-61.

5. Hodis HN, Mack WJ, LaBree L, Selzer RH, Liu CR, Liu $\mathrm{CH}$, et al. The role of carotid arterial intima-media thickness in predicting clinical coronary events. Ann Intern Med 1998; 128: 262-9.

6. Touboul PJ, Vicaut E, Labreuche J, Acevedo M, Torres V, Ramírez-Martínez J, et al. Common carotid artery intima-media thickness: The cardiovascular risk factor multiple evaluation in Latin America (CARMELA) study results. Cerebrovasc Dis 2011; 31: 43-50.

7. Acevedo M, Arnaiz P, Corbalán R, Godoy I, Morales D, Chalhub M, et al. Modificación del grosor intima-media carotídeo según factores de riesgo clásicos y sindrome metabólico con o sin inflamación. Rev Chil Cardiol 2009; 28: 337-48.

8. O'Leary DH, Polak JF, Kronmal RA, Manolio TA, Burke GL, Wolfson SK, Jr. Carotid-artery intima and media thickness as a risk factor for myocardial infarction and stroke in older adults. Cardiovascular Health Study collaborative research group. N Engl J Med 1999; 340: 14-22.

9. Lorenz MW, Markus HS, Bots ML, Rosvall M, Sitzer M. Prediction of clinical cardiovascular events with carotid intima-media thickness: A systematic review and metaanalysis. Circulation 2007; 115: 459-67.

10. Greenland P, Alpert JS, Beller GA, Benjamin EJ, Budoff MJ, Fayad ZA, et al. 2010 ACCF/AHA guideline for assessment of cardiovascular risk in asymptomatic adults: A report of the American College of Cardiology Foundation/American Heart Association Task Force on Practice Guidelines. Circulation 2010; 122: e584-636.

11. Lanas F, Avezum A, Bautista LE, Díaz R, Luna M, Islam $\mathrm{S}$, et al. Risk factors for acute myocardial infarction in Latin America: The INTERHEART Latin American study. Circulation 2007; 115: 1067-74.

12. Lanas F, Potthoff S, Mercadal E, Santíbañez C, Lanas A, Standen D. [individual and population risk in acute 
myocardial infarction: The Chilean INTERHEART study]. Rev Med Chile 2008; 136: 555-60.

13. Pearson TA, Mensah GA, Alexander RW, Anderson JL, Cannon RO III, Criqui M, et al. Markers of inflammation and cardiovascular disease: Application to clinical and public health practice: A statement for healthcare professionals from the Centers for Disease Control and Prevention and the American Heart Association. Circulation 2003; 107: 499-511.

14. Pitt B, Byington RP, Furberg CD, Hunninghake DB, Mancini GB, Miller ME, et al. Effect of amlodipine on the progression of atherosclerosis and the occurrence of clinical events. PREVENT investigators. Circulation 2000; 102: 1503-10.

15. Smilde TJ, van Wissen S, Wollersheim H, Trip MD, Kastelein JJ, Stalenhoef AF. Effect of aggressive versus conventional lipid lowering on atherosclerosis progression in familial hypercholesterolaemia (ASAP): A prospective, randomised, double-blind trial. Lancet 2001; 357: 577-81.

16. Woo KS, Chook P, Yu CW, Sung RY, Qiao M, Leung SS, et al. Effects of diet and exercise on obesity-related vascular dysfunction in children. Circulation 2004; 109: 1981-6.

17. Su T-C, Jeng J-S, Chien K-L, Sung F-C, Hsu H-C, Lee Y-T. Hypertension status is the major determinant of carotid atherosclerosis: A community-based study in Taiwan. Stroke 2001; 32: 2265-71.

18. Lakka TA, Salonen R, Kaplan GA, Salonen JT. Blood pressure and the progression of carotid atherosclerosis in middle-aged men. Hypertension 1999; 34: 51-6.

19. Polak JF, Kronmal RA, Tell GS, O'Leary DH, Savage PJ, Gardin JM, et al. Compensatory increase in common carotid artery diameter. Relation to blood pressure and artery intima-media thickness in older adults. Cardiovascular Health Study. Stroke 1996; 27: 2012-5.

20. Manios E, Tsivgoulis G, Koroboki E, Stamatelopoulos K, Papamichael C, Toumanidis S, et al. Impact of prehypertension on common carotid artery intima-media thickness and left ventricular mass. Stroke 2009; 40:1515-8.

21. Morrison KM, Dyal L, Conner W, Helden E, Newkirk L, Yusuf S, et al. Cardiovascular risk factors and noninvasive assessment of subclinical atherosclerosis in youth. Atherosclerosis 2009.

22. Yan RT, Yan AT, Anderson TJ, Buithieu J, Charbonneau F, Title L, et al. The differential association between various anthropometric indices of obesity and subclinical atherosclerosis. Atherosclerosis 2009; 207: 232-8.

23. 2003 European Society of Hypertension-European Society of Cardiology guidelines for the management of arterial hypertension. J Hypertens 2003; 21: 1011-53.

24. Zanchetti A, Hennig M, Hollweck R, Bond G, Tang R, Cuspidi $\mathrm{C}$, et al. Baseline values but not treatment-in- duced changes in carotid intima-media thickness predict incident cardiovascular events in treated hypertensive patients: Findings in the European Lacidipine Study on Atherosclerosis (ELSA). Circulation 2009; 120: 1084-90.

25. Engstrom G, Melander O, Hedblad B. Carotid intimamedia thickness, systemic inflammation, and incidence of heart failure hospitalizations. Arterioscler Thromb Vasc Biol 2009; 29: 1691-5.

26. Folsom AR, Kronmal RA, Detrano RC, O'Leary DH, Bild DE, Bluemke DA, et al. Coronary artery calcification compared with carotid intima-media thickness in the prediction of cardiovascular disease incidence: The Multi-Ethnic Study of Atherosclerosis (MESA). Arch Intern Med 2008; 168: 1333-9.

27. Burke GL, Bertoni AG, Shea S, Tracy R, Watson KE, Blumenthal RS, et al. The impact of obesity on cardiovascular disease risk factors and subclinical vascular disease: The Multi-Ethnic Study of Atherosclerosis. Arch Intern Med 2008; 168: 928-35.

28. Oren A, Vos LE, Uiterwaal CS, Gorissen WH, Grobbee DE, Bots ML. Change in body mass index from adolescence to young adulthood and increased carotid intimamedia thickness at 28 years of age: The Atherosclerosis Risk in Young Adults Study. Int J Obes Relat Metab Disord 2003; 27: 1383-90.

29. Juonala M, Raitakari M, J SAV, Raitakari OT. Obesity in youth is not an independent predictor of carotid IMT in adulthood. The Cardiovascular Risk in Young Finns Study. Atherosclerosis 2006; 185: 388-93.

30. Sun P, Dwyer KM, Merz CN, Sun W, Johnson CA, Shircore AM, et al. Blood pressure, LDL cholesterol, and intima-media thickness: A test of the "response to injury" hypothesis of atherosclerosis. Arterioscler Thromb Vasc Biol 2000; 20: 2005-10.

31. Hashimoto H, Kitagawa K, Hougaku H, Shimizu Y, Sakaguchi M, Nagai Y, et al. C-reactive protein is an independent predictor of the rate of increase in early carotid atherosclerosis. Circulation 2001; 104: 63-7.

32. Lorenz MW, Karbstein P, Markus HS, Sitzer M. Highsensitivity c-reactive protein is not associated with carotid intima-media progression: The Carotid Atherosclerosis Progression Study. Stroke 2007; 38: 1774-9.

33. Barja S, Acevedo M, Arnaiz P, Berríos X, Bambs C, Guzmán B, et al. [Early markers for atherosclerosis and metabolic syndrome in children]. Rev Med Chile 2009; 137: 522-30.

34. Acevedo M, Arnaiz P, Barja S, Bambs C, Berríos X, Guzmán B, et al. [Relationship of c-reactive protein to adiposity, cardiovascular risk factors and subclinical atherosclerosis in healthy children]. Rev Esp Cardiol 2007; 60: 1051-8. 